\title{
Dengue NS1 Antigen: A Tool in Early Detection of Dengue Virus Infection
}

\author{
RAMA BISWAS ${ }^{1}$, FAZLE RABBI MOHAMMED, ${ }^{2}$ POLY SENGUPTA, ${ }^{1}$ HASAN SHAHREAR AHMED, ${ }^{3}$ \\ MD. MOTLABUR RAHMAN, ${ }^{4}$ MOHAMMAD ABDUS SATTAR SARKER, ${ }^{5}$ MIRZA NAZIMUDDIN ${ }^{6}$
}

\begin{abstract}
:
Objectives: This aim of the study was to evaluate the efficacy of NS1 antigen assay for early diagnosis of dengue virus infection in a tertiary care hospital.

Methods: This cross sectional study was carried out in medicine unit of Square hospital from May to August 20I3. Total 100 patients with dengue fever were included. Complete blood count, alanine aminotransferase (ALT), aspartate aminotransferase (AST), Dengue NS1 antigen and IgM and $\lg G$ antibodies of dengue virus were done in all cases.

Results: Of the 100 sera tested, $75 \%$ were positive for dengue virus infection based on dengue NS1 antigen, IgM antibody and $\lg G$ antibody. Dengue NS1 antigen and $\lg M$, IgG antibody were able to detect dengue virus infection between day 1 to day 8 in $92 \%$ of samples, $86.7 \%$ of samples and $82.6 \%$ of samples respectively. Sixty nine percent (69\%) were found positive for dengue NS1 antigen, $65 \%$ were IgM positive and $62 \%$ were IgG positive. Based on the dengue NS1 antigen and IgM antibody combination, $74 \%$ were positive for dengue virus infections. Sensitivity of Dengue NS1 antigen was $92.3 \%$ and specificity of $74.28 \%$ in comparison to IgM antibody. Based on the antigen and IgG antibody combination, detection rate increased to $75 \%$. Sensitivity of dengue NS1 antigen was $90.3 \%$ and specificity of $65.8 \%$ in comparison to lgG antibody.
\end{abstract}

Conclusion: Dengue NS1 antigen is a useful, sensitive and specific test for early diagnosis of dengue virus infection and it improves diagnostic efficiency in combination with antibody test.

Key words: Dengue fever, NS1 antigen.

\section{Introduction:}

Dengue fever (DF) is the most common arboviral illness in humans. Each year, an estimated 50-100 million cases of dengue fever and 500,000 cases of dengue hemorrhagic fever occur worldwide, with 30000 deaths (mainly in children). Globally $2.5-3$ billion people in approximately 112 tropical and subtropical countries are at risk of dengue infection. ${ }^{1,2}$ In Bangladesh an epidemic of DF broke out in June 2000 when it took heavy toll (5,555 cases and 93 deaths). ${ }^{3,4}$ Due to its impact on high mortality, it is necessary to have a sensitive laboratory assay for early diagnosis of dengue

1. Specialist, Dept. of Medicine, Square Hospitals Ltd, Dhaka.

2. Specialist, Dept. of Respiratory Medicine, Square Hospitals Ltd, Dhaka.

3. Medical Officer, Dept. of Surgery, Bangabandhu Sheikh Mujib Medical University, Dhaka.

4. Assistant Professor, Dept. of Medicine, Dhaka Medical College Hospital.

5. Assistant Professor, Dept. of Medicine, Jessore Medical College Hospital.

6. Consultant, Dept. of Medicine, Square Hopital Ltd, Dhaka.

Correspondence: Dr. Rama Biswas, MBBS, FCPS, Specialist, Department of Medicine, Square Hospitals Ltd,18/F, Bir Uttam Qazi Nuruzzaman Sarak (West Panthapath), Dhaka -1205. E mail: drramabiswas@gmail.com virus infection. The diagnostic methods available are virus isolation, genomic RNA detection by reverse transcriptase PCR and serological test such as immunoglobulin M and G by enzyme linked immunosorbent assay (ELISA). The first two assays need specialized laboratory as well as well trained laboratory personnel which is not widely available. So dengue specific antibody test is the most commonly available method. ${ }^{5}$ IgM antibodies are detectable approximately 46 days after onset of fever. By day 5 , it became detectable in $80 \%$ cases and $93-99 \%$ cases by day 6 to 10 which delay in early diagnosis of dengue infection. ${ }^{6}$ Dengue virus nonstructural protein 1(NS1) antigen, a highly structured glycoprotein, produced both in membrane associated and secretory forms is detectable in blood from first day after onset of fever up to day $9 .^{7}$ So detection of dengue NS1 antigen represents a new approach to the diagnosis of acute dengue virus (DV) infection. In our study we aimed to evaluate the NS1 antigen assay for early diagnosis of dengue virus infection in a tertiary care hospital.

\section{Materials and Methods:}

This cross sectional study was carried out in medicine unit of Square Hospital from May to August 2013. Total 100 patients with DF were included. DF was defined according 
to National guideline of Bangladesh. The demographic profile was taken from each case. Complete blood count, alanine aminotransferase (ALT), aspartate aminotransferase (AST), Dengue NS1 antigen and IgM and IgG antibodies of dengue virus were done in all cases. Other relevant investigations were done to determine complications or to exclude differential diagnosis when required.

\section{Results:}

In this study, total 100 patients with DF were included with male, female ratio 1:0.8. Majority of the cases $(86 \%)$ were below 50 years of age. About 30\% cases were students and $22 \%$ were service holders. More than $50 \%$ cases came from Dhanmondi area. Total $85 \%$ patients were classical dengue fever and $15 \%$ of dengue hemorrhagic fever. Only $2 \%$ complications were noted and there were no adverse outcome.

Leucopenia was noted in 59\% cases and mean WBC was $3677.88 \pm 1382.05 \mathrm{SD}$. Total platelet count was found $<100,000 /$ $\mathrm{cmm}$ in all patients and it was $<10,000 / \mathrm{cmm}$ in $12 \%$ patients. Mean ALT of the study population was $168.43 \pm 213.12$ SD and mean AST was 213.86+322.62. Dengue NS1 antigen was positive in $69 \%$ cases. IgM antibody to dengue virus was positive in $65 \%$ cases and $\operatorname{IgG}$ in $62 \%$ cases.

Of the 75 dengue positive samples, dengue NS1 antigen and IgM, IgG antibody were able to detect dengue virus infection between day 1 to day 8 in $92 \%$ of samples $(69 / 75), 86.7 \%$ of samples (65/75) and $82.6 \%(62 / 75)$ of samples respectively.

Combination of Dengue NS1 antigen and antibody tests:

The results of Dengue NS1 antigen were compared to the results of dengue antibody tests (IgM and $\operatorname{IgG}$ ). Total 69 samples were positive for IgM, giving the serological test a detection rate of $69 \%$. Based on the combination of dengue NS1 antigen and IgM antibody test, total 74 patients (74\%) were positive for dengue virus infections. Dengue NS1 antigen was not detected in $7.69 \%(5 / 65)$ of $\operatorname{IgM}$ positive samples but it was detected in $25.7 \%$ (9/35) of IgM negative samples. So sensitivity of Dengue NS1 antigen was $92.3 \%$ and specificity of $74.28 \%$ in comparison to $\operatorname{IgM}$ antibody.

Table I

Comparison of Dengue NS1 antigen and dengue antibody $\operatorname{Ig} M$

\begin{tabular}{llcc}
\hline & & \multicolumn{2}{c}{ Dengue antibody (IgM) } \\
\cline { 3 - 4 } & $\begin{array}{c}\text { Positive } \\
(\mathrm{n}=65)\end{array}$ & $\begin{array}{c}\text { Negative } \\
(\mathrm{n}=35)\end{array}$ \\
\hline Dengue NS1 & Positive $(\mathrm{n}=69)$ & 60 & 9 \\
antigen & Negative $(\mathrm{n}=31)$ & 5 & 26 \\
\hline
\end{tabular}

Dengue NS1 antigen was also compared to dengue antibody IgG. A total of $62 \%$ samples were positive giving detection rate of $62 \%$. Based on the antigen and $\mathrm{IgG}$ antibody combination, detection rate increased to $75 \%$. Dengue NS1 antigen was not detected in $9.6 \%(6 / 62)$ of $\mathrm{IgG}$ positive samples but detected in 34.2\% (13/38) of negative samples. So sensitivity of dengue NS1 antigen was $90.3 \%$ and specificity of $65.8 \%$ in comparison to IgG antibody.

Table-II

Comparison of Dengue NS1 antigen and dengue antibody $\operatorname{Ig} G$

\begin{tabular}{llcc}
\hline & & \multicolumn{2}{c}{ Dengue antibody (IgG) } \\
\cline { 3 - 4 } & $\begin{array}{c}\text { Positive } \\
(\mathrm{n}=62)\end{array}$ & $\begin{array}{c}\text { Negative } \\
(\mathrm{n}=38)\end{array}$ \\
\hline Dengue NS1 & Positive $(\mathrm{n}=69)$ & 56 & 13 \\
antigen & Negative $(\mathrm{n}=31)$ & 6 & 25 \\
\hline
\end{tabular}

\section{Discussion:}

With the escalating incidence of dengue and the absence of vaccine for prevention of the disease, it is important to detect early dengue virus (DENV) infection for management of patients as well as for effective public health control of dengue outbreaks. Various studies have confirmed the detection of dengue NS1 antigen is useful for early diagnosis of dengue infections. ${ }^{8,9}$ Of the 100 patients tested, $69 \%$ patients were positive on dengue NS1 antigen, 65\% were IgM positive and $62 \%$ were IgG positive. Serological testing has always been the method of choice in most laboratory settings. In this study, the detection of DENV antibodies, IgM and /or IgG antibody tests, were found in 67\% cases. Combination of antigen test with antibody were analysed to determine the performance in detecting DENV infections. Based on the combination of dengue NS1 antigen and IgM, total 74 patients $(74 \%)$ were positive for dengue virus infections. Dengue NS1 antigen was not detected in $7.69 \%$ (5/65) of IgM positive samples but it was detected in $25.7 \%$ (9/35) of IgM negative samples. So sensitivity of Dengue NS1 antigen was $92.3 \%$ and specificity of $74.28 \%$ in comparison to IgM antibody. Based on the antigen and IgG antibody combination, detection rate increased to $75 \%$. Dengue NS1 antigen was not detected in 9.6\% (6/62) of IgG positive samples but detected in $34.2 \%$ (13/38) of negative samples. So sensitivity of dengue NS1 antigen was $90.3 \%$ and specificity of $65.8 \%$ in comparison to IgG antibody. So the combination of antigen and antibody test gave a better yield for detecting dengue infections. A study conducted by Kassim et.al in 2011 was analyzed which revealed that 
sensitivity of dengue NS1 antigen was $42.05 \%$ and specificity of $78.21 \%$ when compared to dengue antibody. ${ }^{10}$

Dengue NS1 antigen has allowed for early detection of DENV infection as the antigens remain detectable in blood for 5 days after onset of fever and rapidly disappear after formation of specific antibodies. ${ }^{11}$ In this study, DENV antigens were detected as early as day 1 to day 8 of fever. It is comparable to a study by Alcon et al published in 2006 who recovered NS1 antigen up to day 9 of symptoms. ${ }^{12}$ A study conducted by Koraka et al in 2003 showed that antigen detection was low in endemic area due to formation of immune complexes following a secondary dengue infection and inappropriate temperature storage of samples. ${ }^{13}$ So it is important that samples are collected, stored and transported properly to maintain the quality and stability of samples tested.

Dengue NS1 antigen assay is simple and easily to perform especially in laboratories that are already performing ELISA. Within a few hours, the physician can detect DENV infection in outpatient clinic and early detection will enable was not only in patient management but also notify public health authorities. However, it was unable to distinguish between serotype of dengue virus which was possible by PCR. As most laboratories have limited funds to set up PCR tests, NS1 antigen should be considered as an additional diagnostic tool for early dengue virus infection. The combination of antigen and antibody tests may increase the diagnostic efficiency on DENV infection. This is in comparable with Shekaran et al in 2007 who showed combination of tests were sensitive and specific in endemic settings. $^{14}$

\section{Conclusion:}

The evaluation of Dengue NS1 antigen assay shows that it is useful, sensitive and specific test for early diagnosis of dengue virus infection in the laboratories that have limited resources, lack viral culture or RT-PCR facilities.

\section{Conflict of Interest: None}

\section{References:}

1. Smith AW, Chen LH, Massad E, Wilson ME. Threat of dengue to blood safety in dengue-endemic countries. Emerg Infect Dis 2009; 15:8-11.

2. Guzman MG, Kouri G. Dengue: an update. Lancet Infect Dis 2002; $2: 33-42$.
3. ICDDRB Annual report, 2001. Bangladesh Dengue website. In: http://www.geocities.com/prevent-dengue/. Accessed 16 September, 2013

4. Aziz MA, Graham RR, Gregg MB. "Dacca fever" An outbreak of dengue. Pak j Med Res 1967; 6: 83-92.

5. Chakravarti A, Kumaria R, Batra VV, Verma V. Improved detection of dengue virus serotypes from serum samplesEvaluation of single tube multiplex RT-PCR with cell culture. Dengue Bulletin 2006; 30:133-40.

6. Guzman MG, Kouri G. Dengue diagnosis, advances and challenges. Int J Infect Dis 2004; 8: 69-80.

7. Young PR, Hilditch PA, Bletchly C, Halloran W. An antigen capture enzyme-linked immunosorbent assay reveals high levels of the dengue virus protein NS1 in the sera of infected patients. J Clin Microbiol 2000;38: 1053-7.

8. Ramirez AH, Moros Z, Comach G, Zambrano J, Bravo L, Pinto B et al. Evaluation of dengue NS1 antigen detection tests with acute sera from patients infected with dengue virus in Venezuela. Dia Gn Microbiol Infect Dis 2009; 65: 247-53.

9. Zainah S, Abdul Wahab AH, Mariam M, Fauziah MK, Khairul AH, Roslina I et al. Performance of a commercial rapid dengue NS1 antigen immunochromatography test with reference to dengue NS1 antigen-capture ELISA. J Virol Methods 2009; 155:157-60.

10. Kassim FM, Izati MN, TgRogayah TAR, Apandi YM, Saat $Z$. Use of dengue NS1 antigen for early diagnosis of dengue virus infection. Southeast Asian J Trop Med Public Health 2011; 42 (3): 562-569

11. Kao CL, King CC, Chao DY, Wu HL, Chang GJJ. Laboratory diagnosis of dengue virus infection: current and future perspectives in clinical diagnosis and public health. $\mathrm{J}$ Microbiol Immonol Infect 2005; 38:5-16.

12. Alcon S, Sivard P, Drouet MT, Talarmin A, Rice C, Flamand M. Secretion of flavi viral nonstructural protein NS1: from diagnosis to pathogenesis. In: New treatment strategies for dengue and other flavi viral diseases. Chichester: Wiley; 2006. p. 233-50.

13. Koraka P, Burghoorn-Mass CP, Falconar A, Setiati TE, Djamiatun K, Groen J et al. Detection of immune-complexdissociated nonstructural protein 1 antigen in patients with acute dengue virus infections. J Clin Microbiol 1993;41: 4154-9.

14. Sekaran SD, Lan EC, Mahesawarappa KB, Appanna R, Subramaniam G. Evaluation of Dengue NS1 capture ELISA assay for the rapid detection of dengue. J Infect Develop Countries 2007; 1:182-8. 